\title{
Potencial ornamental de Baccharis milleflora e Baccharis tridentata como folhagem de corte
}

\author{
Ornamental potential use of Baccharis milleflora and \\ Baccharis tridentata as ornamental cut foliage
}

Grasiela Bruzamarello Tognon ${ }^{\mathrm{I}^{*}}$ Francine Lorena Cuquel $^{\mathrm{I}}$

\section{RESUMO}

A prospecção de novas plantas ornamentais a partir de espécies nativas amplia a oferta de produtos para o mercado da floricultura. Entretanto, antes de uma espécie ser indicada como ornamental, suas características estéticas devem ser avaliadas pelo público consumidor. Com isso, o objetivo deste estudo foi prospectar novas plantas para uso como folhagem de corte e avaliar o seu potencial ornamental. O potencial ornamental das espécies prospectadas foi avaliado por 10 profissionais especialistas em arte floral, com base em metodologia que considera características qualitativas e quantitativas de importância ornamental para o mercado consumidor. Cada critério foi pontuado por meio de notas de dez (mínima) a 100 (máxima). Baccharis milleflora e Baccharis tridentata foram selecionadas na região de Mata Atlântica, em borda de mata, por apresentarem ocorrência frequente na paisagem, hastes longas, folhas exuberantes, com coloração destacada na paisagem e aspecto aparentemente rústico. Ambas as espécies apresentam alto potencial ornamental para uso como folhagem de corte com características adequadas para o uso como complementos para arranjos florais.

Palavras-chave: Asteraceae, análise sensorial, arranjo floral, planta nativa, prospecção.

\section{ABSTRACT}

Identification of new ornamental plants among native species widens the offer of new products for the floricultural market. However, before indicating the use of a species as potentially ornamental, evaluation of its aesthetic features by possible consumers is of great importance. Thus, the aims of this study were identification and evaluation of the ornamental potential of new plants as cut foliage. The ornamental potential of species was determined based on the methodology that considers qualitative and quantitative characteristics of ornamental importance for the consumer market. Each criterion was assessed by scores from ten (minimum) to 100 (maximum). Baccharis milleflora and Baccharis tridentata were selected in the Atlantic Forest, region of forest edge, because they has frequent occurrence in the landscape, long stems, lush leaves, with outstanding color in the landscape and rustic aspect apparently. These two species showed high potential to be used as ornamental cut foliage, confirming adequate performance as complementary features in floral arrangements.

Key words: Asteraceae, sensory analysis, floral arrangement, native plants, prospecting.

\section{INTRODUÇÃO}

A floricultura é um mercado bastante exigente e em constante busca por novos produtos (BOUMAZA et al., 2010). Entretanto, mesmo com a enorme biodiversidade brasileira com potencial para uso ornamental, a prospecção da flora nativapara este fim ainda se encontra bastante incipiente (STUMPF et al., 2008). Além disso, para definição de uma planta como ornamental são necessárias avaliações de suas características estéticas, que preferencialmente devem ser realizadas por profissionais ligados à floricultura (HEIDEN et al., 2006; BOUMAZA et al., 2009; BOUMAZA et al., 2010).

No mercado da floricultura, um segmento de destaque é o das flores e folhagens de corte. As folhagens de corte são utilizadas para dar sustentação e preenchimento, melhorando a aparência dos arranjos florais (BASKETT \& SMITH, 2006; LOBO-

IPrograma de Pós-graduação em Agronomia, Produção Vegetal, Departamento de Fitotecnica e Fitossanitarismo, Universidade Federal do Paraná (UFPR), Rua dos Funcionários, 1540, CP 19061, 80035-050, Juvevê, PR, Brasil. E-mail: gbtbio@gmail.com. *Autor para correspondência. 
GUERRERO, 2009). As folhagens a serem utilizadas para confecção de arranjos são escolhidas com base no tamanho, na forma e no dinamismo que irão conferir ao arranjo floral, podendo se mesclar mais de uma folhagem em uma mesma combinação (SCACE, 2001).

Atualmente, as folhagens mais comumente utilizadas em arranjos florais são: antúrio (Anthurium sp.), aspargo-samambaia (Asparagus setaceus), camélia (Camellia japonica), cordelines (Cordyline terminalis), dracena (Dracena sp.), eucalipto (Eucalyptus cinerea), junco (Juncus effusus), pittosporum (Pittosporum undulatum), podocarpos (Podocarpus sp.) e ruscus (Ruscus aculeatus) (LOBO-GUERRERO, 2009). Dentre essas espécies comercializadas, a maioria é exótica e demanda alta tecnificação para produção, inviabilizando o cultivo por pequenos produtores sem infraestrutura de produção, sendo a produção de espécies nativas rústicas uma vantagem.

Para suprir a demanda do mercado e fazer o resgate de espécies nativas, objetivou-se, com este trabalho, prospectar e avaliar o potencial ornamental de duas espécies nativas para serem utilizadas como folhagem de corte em arranjos florais.

\section{MATERIAL E MÉTODOS}

Prospecção de espécies com potencial ornamental Para realização da prospecção das espécies com potencial ornamental para uso como folhagem de corte, foram realizadas expedições a campo mensalmente entre os anos de 2012 e 2013 em regiões de Mata Atlântica, em borda de mata, no entorno dos Municípios de Piraquara e Quatro-Barras, no Estado do Paraná (S 2530.527'; W 4902.225'; altitude de $818 \mathrm{~m})$. A região apresenta classificação climática, de acordo com Köppen, do tipo $\mathrm{Cfb}$, a temperatura média anual é de $17-18^{\circ} \mathrm{C}$, com precipitação média anual de 1200-1400mm, sem estação seca definida (IAPAR, 2014). Durante a prospecção, foram estabelecidos os seguintes critérios visuais para seleção: exuberância das folhas, hastes longas (acima de $40 \mathrm{~cm}$ ), coloração verde brilhante, que se destaque na paisagem, aspecto aparentemente rústico e ocorrência frequente da espécie na região de estudo.

\section{Coleta e identificação do material vegetal}

Hastes de duas espécies consideradas promissoras foram coletadas no município de Piraquara - PR (S 2530.527'; W 4902.225'; altitude de $818 \mathrm{~m}$ ) em março de 2013. Para ambas as espécies, logo após a coleta o material vegetal, foi acondicionado em recipientes plásticos de $20 \mathrm{~L}$ com água e imediatamente transportado ao laboratório para condução dos experimentos. Também foram preparadas exsicatas para identificação das duas espécies, sendo depositadas no Herbário das Faculdades Integradas Espíritas, sob número de inscrição HFIE 9.125 para B. milleflora e HFIE 9.126 para $\boldsymbol{B}$. tridentata.

\section{Avaliação do potencial ornamental}

A caracterização morfológica das espécies foi realizada por meio das seguintes avaliações: comprimento das hastes $(\mathrm{cm})$, diâmetro das hastes (mm) e número de ramificações principais/haste. $\mathrm{O}$ potencial ornamental de duas espécies selecionadas foi determinado com base em metodologia que considera características qualitativas e quantitativas de importância ornamental para o mercado consumidor. Hastes dessas espécies foram submetidas à avaliação sensorial por 10 profissionais especialistas em arte floral (que corresponde a 50\% das floriculturas mais representativas de Curitiba-PR), juntamente com a planilha para avaliação dos seguintes critérios: comprimento, diâmetro, flexibilidade, forma e volume da haste, cor e brilho das folhas e aroma. Cada critério foi pontuado por meio de notas de dez (mínima) a 100 (máxima). Assim, o potencial ornamental foi estabelecido pela média das notas atribuídas para cada critério nas seguintes categorias: A) acima de 70 pontos: potencial ornamental elevado; B) de 50 até 69 pontos: potencial ornamental médio; C) de 25 até 49 pontos: potencial ornamental baixo; D) menor que 25: potencial ornamental mínimo (adaptada de STUMPF et al., 2007).

Para determinar as alternativas de uso na arte floral das duas espécies, foram anexadas à planilha de avaliação, as alternativas de uso: ikebana, bouquet, arranjo de mesa e arranjo para eventos. Assim cada avaliador marcou a (s) alternativa (s) em que a espécie pode ser utilizada. Esses resultados foram expressos em porcentagem para cada alternativa de uso.

\section{RESULTADOS E DISCUSSÃO}

$\mathrm{Na}$ prospecção de plantas nativas com potencial para uso como folhagem de corte, as espécies Baccharis milleflora DC. e Baccharis tridentata Vahl foram selecionadas por apresentarem os aspectos visuais requeridos e por se destacarem das demais espécies ocorrentes na região em estudo. Além disso, foi observado que $\boldsymbol{B}$. milleflora e $\boldsymbol{B}$. tridentata são plantas frequentes na paisagem local, mostrando-se adaptadas às condições climáticas da região. Ambas as espécies apresentam hastes 
longas e a coloração exuberante manteve-se ao longo dos meses do ano nos quais foram realizadas as expedições a campo.

B. milleflora pertence à família Asteraceae, conhecida como carqueja (BUDEL et al., 2005), é uma espécie arbustiva e endêmica do Brasil que aprecia substratos rupícolas e terrícolas, com distribuição geográfica na região Sudeste e Sul. A espécie B. tridentata, também da família Asteraceae e nativa do Brasil, é conhecida popularmente como vassoura ou carqueja-folhuda (BUDEL et al., 2005), apresenta folhas inteiras, com margem do limbo recortada e filotaxia alterna. Sua distribuição geográfica de ocorrência é no Centro-Oeste, Sudeste e Sul (HEIDEN \& SCHNEIDER, 2014).

As duas espécies mostram aspecto de rusticidade, o que pode ser uma vantagem para seu cultivo, sem que haja necessidade de cultivo protegido ou alta tecnologia para produção, tornado- se uma alternativa para o segmento da floricultura que não dispõe de infraestrutura. Atualmente, muitas das folhagens de corte são provenientes do extrativismo, como a guaricanga (Geonoma pohliana) (OSHIRO et al., 2001) e a samambaia (Rhumora adiantiformis) (RIBAS \& MIGUEL, 2004), ou de produtores mais tecnificados e comercializadas pelo Veiling Holambra, como o ruscus (Ruscus aculeatus) e o Pitósporo (Pittosporum tobira) (IBRAFLOR, 2014), restando pouca ou nenhuma opção de produção para pequenos produtores.

Com relação aos aspectos estéticos, o volume e a forma das hastes são importantes no momento da escolha do estilo do arranjo a ser confeccionado (NAEVE \& MIDCAP, 2007). Hastes de B. milleflora e B. tridentata (Figura 1) apresentaram acima de 70 pontos nesses critérios (Tabela 1), entretanto, o volume, que está relacionado ao número de ramificações, no caso de $\boldsymbol{B}$. milleflora

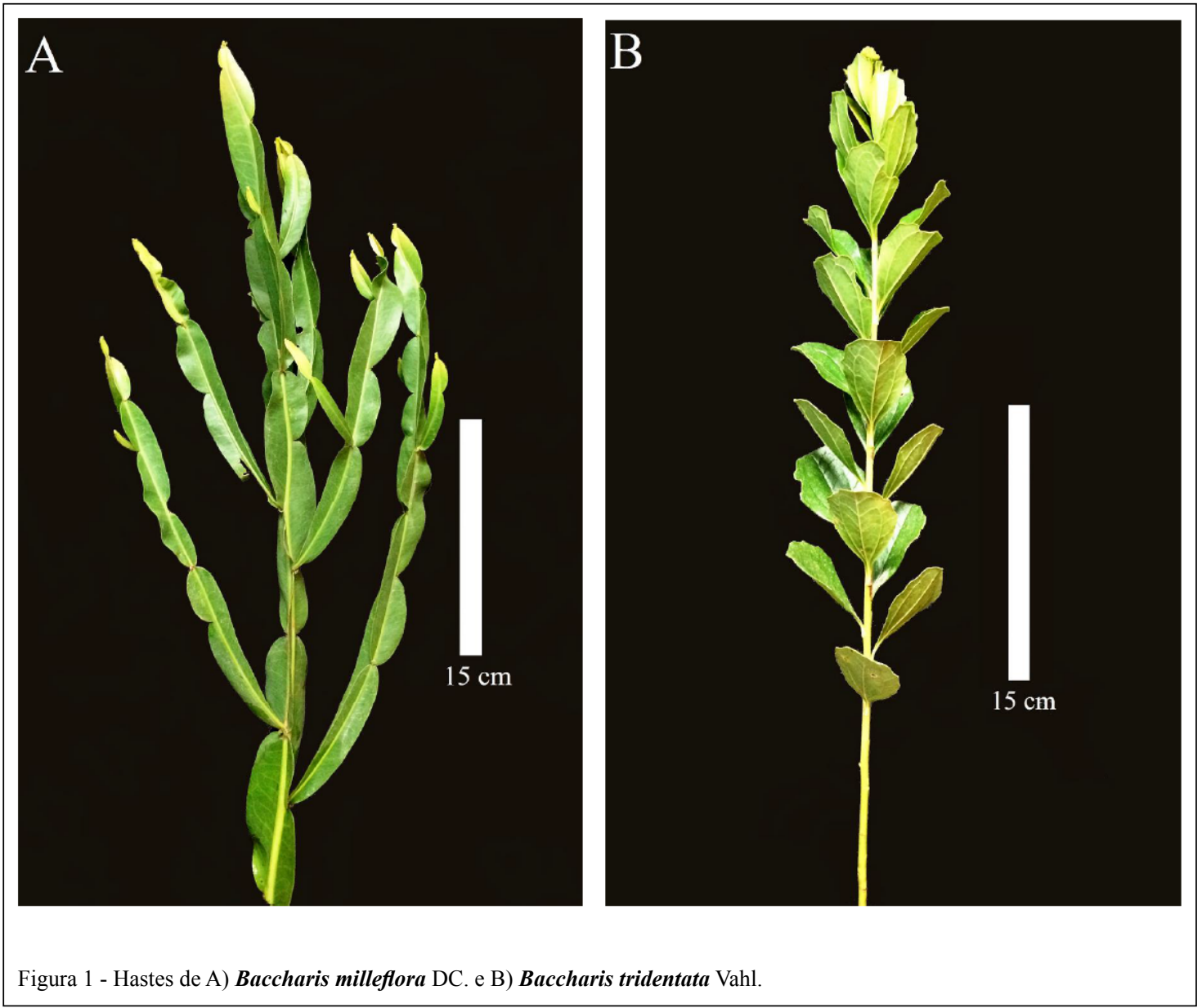

Ciência Rural, v.46, n.1, jan, 2016. 
Tabela 1 - Média das notas atribuídas por 10 avaliadores, especialistas em arranjos florais, para as características de interesse ornamental das hastes de Baccharis milleflora DC. e Baccharis tridentata Vahl.

\begin{tabular}{|c|c|c|c|c|c|c|c|c|c|c|c|}
\hline \multirow{2}{*}{ Características } & \multicolumn{11}{|c|}{-------------------------------------------Notas atribuídas por cada avaliador---------------------------------------- } \\
\hline & 1 & 2 & 3 & 4 & 5 & 6 & 7 & 8 & 9 & 10 & Médias \\
\hline Comprimento & 70 & 80 & 70 & 80 & 80 & 70 & 100 & 80 & 100 & 70 & 80 \\
\hline Diâmetro & 90 & 70 & 70 & 100 & 80 & 90 & 100 & 50 & 100 & 90 & 84 \\
\hline Flexibilidade & 90 & 50 & 70 & 100 & 90 & 100 & 70 & 70 & 70 & 70 & 78 \\
\hline Forma da haste & 90 & 80 & 70 & 80 & 100 & 100 & 70 & 90 & 80 & 80 & 84 \\
\hline Volume da haste & 70 & 80 & 70 & 80 & 80 & 90 & 70 & 80 & 70 & 80 & 77 \\
\hline Cor das folhas & 90 & 70 & 100 & 70 & 90 & 100 & 80 & 40 & 80 & 90 & 81 \\
\hline Brilho das folhas & 80 & 60 & 60 & 100 & 90 & 100 & 60 & 70 & 100 & 100 & 82 \\
\hline Aroma & 90 & 60 & 100 & 100 & 90 & 100 & 70 & 60 & 100 & 100 & 87 \\
\hline Média geral & & & & & & & & & & & $82^{*}$ \\
\hline \multirow{2}{*}{ Características } & & & & & tas at & das & ada av & dor-- & & 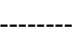 & ------ \\
\hline & 1 & 2 & 3 & 4 & 5 & 6 & 7 & 8 & 9 & 10 & Médias \\
\hline Comprimento & 90 & 90 & 70 & 80 & 100 & 70 & 80 & 70 & 80 & 70 & 79 \\
\hline Diâmetro & 100 & 100 & 90 & 80 & 80 & 90 & 100 & 80 & 100 & 100 & 92 \\
\hline Flexibilidade & 100 & 100 & 90 & 80 & 70 & 90 & 100 & 80 & 90 & 80 & 88 \\
\hline Forma da haste & 100 & 90 & 80 & 90 & 100 & 100 & 80 & 80 & 100 & 90 & 91 \\
\hline Volume da haste & 100 & 100 & 90 & 80 & 100 & 100 & 100 & 90 & 90 & 80 & 93 \\
\hline Cor das folhas & 100 & 100 & 90 & 100 & 100 & 100 & 100 & 90 & 80 & 100 & 96 \\
\hline Brilho das folhas & 100 & 100 & 70 & 100 & 100 & 80 & 90 & 100 & 100 & 90 & 93 \\
\hline Aroma & 100 & 100 & 90 & 90 & 100 & 100 & 100 & 100 & 90 & 100 & 97 \\
\hline Média geral & & & & & & & & & & & $91^{*}$ \\
\hline
\end{tabular}

*Acima de 70 pontos: potencial ornamental elevado; de 50 até 69 pontos: potencial ornamental médio; de 25 até 49 pontos: potencia] ornamental baixo; menor que 25: potencial ornamental mínimo.

de $7 \pm 2$ e de $\boldsymbol{B}$. tridentata, que não apresenta ramificações, poderia ser uma característica a ser melhorada (por meio de seleção natural de plantas na paisagem) para utilização em diferentes estilos de arranjos florais. Contudo, na forma como se apresentam, as hastes de ambas as espécies foram utilizadas para elaboração de arranjos lineares cheios (line-mass arrangements) (Figura 2). Esse estilo de arranjo combina as influências japonesa e europeia, apresenta forma vertical, assimétrica e organizada, e é feito para ser visto de frente (HONEYWELL \& CULBERT, 2005).

O comprimento das hastes de $\boldsymbol{B}$. milleflora $(65 \pm 4 \mathrm{~cm})$ e $\boldsymbol{B}$. tridentata $(69 \pm 2 \mathrm{~cm})$ apresentou média de avaliação pelos floristas de 80 e 79, respectivamente (Tabela 1), isso provavelmente porque propiciam maiores possibilidades de uso destas folhagens tanto em arranjos maiores quanto em arranjos de pequeno porte, podendo ser uma opção para acompanhar flores tropicais como Strelitzia reginae ou Heliconia sp., que apresentam hastes mais longas. Além disso, pelas normas de qualidade estabelecidas pelo Veiling
Holambra, hastes de folhagens ornamentais devem apresentar entre 40 e $100 \mathrm{~cm}$ de comprimento, sendo B. milleflora e B. tridentata enquadradas na Classe 60 (IBRAFLOR, 2014), o que as coloca dentro do padrão exigido pelo mercado.

Segundo NAEVE \& MIDCAP (2007), a flexibilidade das hastes é uma característica importante para a confecção de arranjos florais, sendo o diâmetro da haste o caráter que confere a maior ou menor flexibilidade. O diâmetro médio observado foi de $6,8 \pm 1,2 \mathrm{~mm}$ para $\boldsymbol{B}$. milleflora e $7,1 \pm 0,98 \mathrm{~mm}$ para $\boldsymbol{B}$. tridentata. As hastes de $\boldsymbol{B}$. milleflora tiveram maior heterogeneidade na sua flexibilidade, ou seja, algumas hastes mostraram-se mais flexíveis que outras, fato que se comprova pela avaliação dos floristas (Tabela 1). Essa heterogeneidade pode estar relacionada ao ponto de maturação da planta ou à variabilidade genética presente em espécies não melhoradas, entretanto pode ser um ponto positivo, pois amplia a sua utilização em diferentes tipos de arranjos. Além disso, devido às características das hastes de ambas as espécies, a necessidade de 

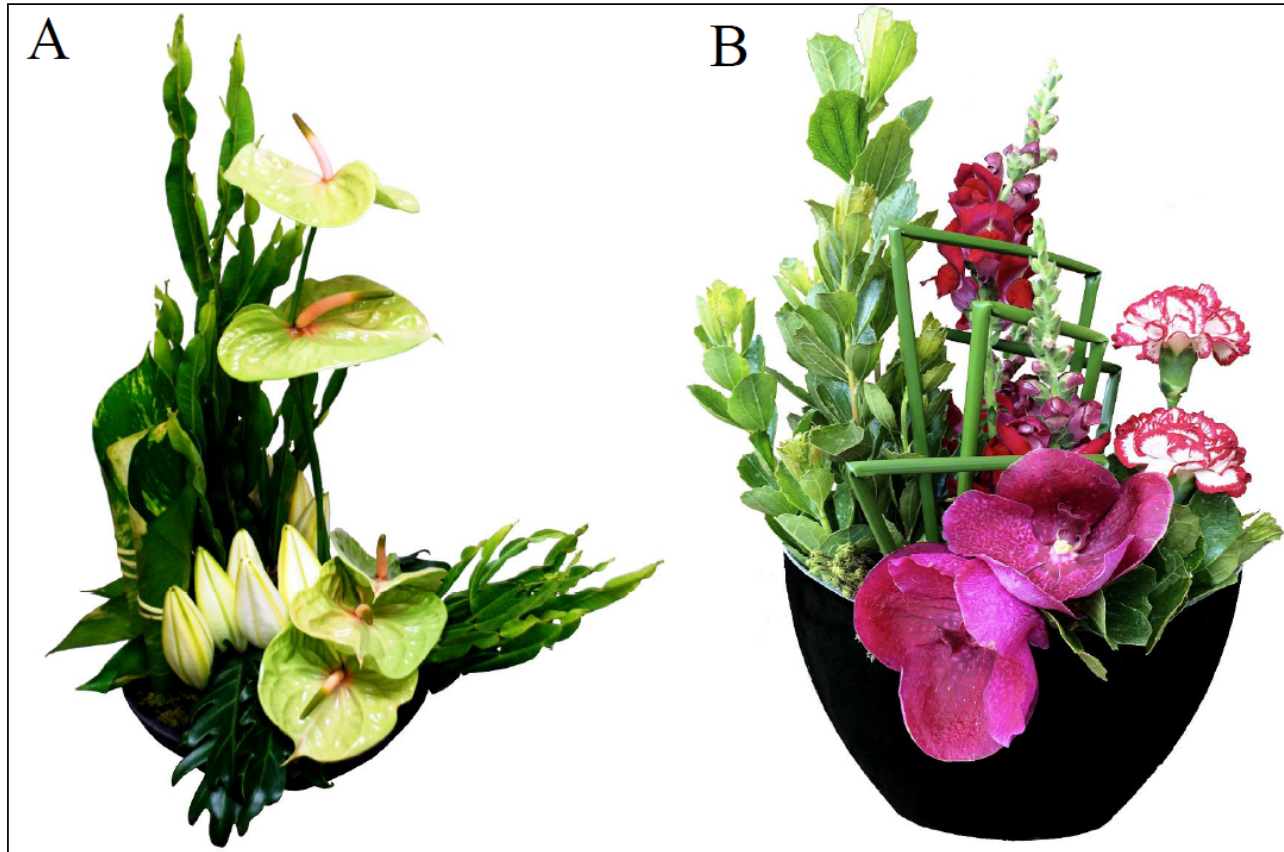

Figura 2 - Arranjo estilo linear cheio (line-mass arrangements) confeccionado com hastes de A) Baccharis milleflora DC. e B) Baccharis tridentata Vahl.

reforço ou aramação é dispensada (KIKUCHI, 1995), facilitando a utilização, armazenamento e transporte.

A coloração é uma característica de grande importância na composição de arranjos florais (HONEYWELL \& CULBERT, 2005; NAEVE \& MIDCAP, 2007), ambas as hastes de B. milleflora e de $\boldsymbol{B}$. tridentata apresentaram avaliação positiva para brilho e cor (Tabela 1). Com isso, supõe-se que a coloração verde brilhante dessas espécies possa fornecer a característica de leveza conferida pelas folhagens verdes aos arranjos (HONEYWELL \& CULBERT, 2005). Além do mais, segundo relato dos avaliadores, as folhas brilhosas características de $\boldsymbol{B}$. tridentata dispensam o uso de produtos a base de cera de carnaúba, comumente utilizados para dar brilho a folhagens de corte tradicionalmente comercializadas.

As hastes de $\boldsymbol{B}$. milleflora e $\boldsymbol{B}$. tridentata não apresentam forte aroma. Para os avaliadores, esse critério foi considerado favorável (Tabela 1), pois, segundo TOGNON et al. (2015), os floristas buscam trabalhar com folhagens de corte sem aroma para que não haja interferência na fragrância exalada pelas flores que farão parte do arranjo.

Segundo 78\% dos avaliadores, hastes de B. milleflora foram consideradas adequadas para uso em arranjos para eventos e ikebanas, $89 \%$ sugeriram
B. milleflora para compor arranjos de mesa e $56 \%$ indicaram seu uso para bouquet. Já as hastes de $\boldsymbol{B}$. tridentata, segundo $100 \%$ dos avaliadores, podem ser utilizadas para confecção de ikebana e para arranjos de mesa, $80 \%$ dos avaliadores indicaram para uso em bouquet e $40 \%$ dos avaliadores sugeriram seu uso para compor arranjos para eventos.

Assim, como outras espécies do gênero Baccharis, como por exemplo, B. articulata, $\boldsymbol{B}$. usterii (STUMPF et al., 2008), B. angustifolia, $\boldsymbol{B}$. glomeruliflora, B. neglecta (BAILEY \& BAILEY, 1976) e B. uncinela (TOGNON et al., 2015), as espécies $\boldsymbol{B}$. milleflora e $\boldsymbol{B}$. tridentata foram classificadas como de alto potencial ornamental (Tabela 1), de acordo com as características apresentadas e avaliadas pelos profissionais da floricultura. Dessa forma, estas espécies surgem como alternativas para substituir outras espécies exóticas usadas como folhagem de corte, como ruscus (Ruscus aculeatus) e camélia (Camellia japonica), e principalmente aquelas que são alvo de extrativismo, como, por exemplo, a guaricanga (Geonoma pohliana) (OSHIRO et al., 2001) e a samambaia (Rumohra adiantiformis) (RIBAS \& MIGUEL, 2004). Entretanto, estudos sobre a durabilidade pós-colheita ainda não foram 
realizados. Em vista disso, recomendam-se estudos relacionados à sua senescência após a colheita e manejos adequados para prolongar a vida de vaso dessas espécies, além de estudos voltados à sua propagação e manejo de produção.

\section{CONCLUSÃO}

As espécies selecionadas foram Baccharis milleflora e Baccharis tridentata. Ambas as espécies apresentam alto potencial ornamental para uso como folhagem de corte, apresentando características adequadas para o uso, como complementos, em ikebana, bouquet, arranjo de mesa e arranjo para eventos.

\section{AGRADECIMENTOS}

À Coordenação de Aperfeiçoamento de Pessoal de Nível Superior (CAPES) pela bolsa de doutorado concedida ao primeiro autor.

\section{REFERÊNCIAS}

BAILEY, E.; BAILEY, L. Hortus third. New York: MacMillan, 1976. $1312 \mathrm{p}$.

BASKETT, M.; SMITH, E. Classic floral designs. New York: Sterling Publishing, 2006. 128p.

BOUMAZA, R. et al. Visual characterization of the esthetic quality of the rosebush. Journal of Sensory Studies, v.24, p.774796, 2009. Disponível em: <http://onlinelibrary.wiley.com/ doi/10.1111/j.1745-459X.2009.00238.x/pdf>. Acesso em: 20 jan. 2015. doi: 10.1111/j.1745-459X.2009.00238.x.

BOUMAZA, R. et al. Sensory profiles and preference analysis in ornamental horticulture: The case of the rosebush. Food Quality and Preference, v.21, n.8, p.987-997,2010. Disponível em: $<\mathrm{http}: / /$ www.sciencedirect.com/science/article/pii/S0950329310000777> Acesso em: 20 jan. 2015. doi: 10.1016/j.foodqual.2010.05.003.

BUDEL J.M. et al. O progresso da pesquisa sobre o gênero Baccharis, Asteraceae: I - Estudos botânicos. Revista Brasileira de Farmacognosia, v.15, n.3, p.268-271, 2005. Disponível em: $<$ http://dx.doi.org/10.1590/S0102-695X2005000300018>. Acesso em: 20 jan. 2015. doi: 10.1590/S0102-695X2005000300018.

HEIDEN, G. et al. Considerações sobre o uso de plantas ornamentais nativas. Revista Brasileira de Horticultura Ornamental, v.12, n.1, p.2-7, 2006. Disponível em: <http://132.248.9.34/hevila/ Revistabrasileiradehorticulturaornamental/2006/vol12/no1/1. pdf>. Acesso em: 20 jan. 2015.

HEIDEN, G.; SCHNEIDER, A. Baccharis in lista de espécies da flora do Brasil. Jardim Botânico do Rio de Janeiro, 2014.
Disponível em: < http://floradobrasil.jbrj.gov.br/jabot/floradobrasil/ FB5257>. Acesso em: 20 jan. 2015.

HONEYWELL, E.R.; CULBERT, J.R. Principles of floral arrangement - Baxter County, 2005. Disponível em: <http:// baxtercountyfair.org/pdf/Principles_of_Floral_Arrangement.pdf $>$. Acesso em: 20 jan. 2015.

IAPAR (INSTITUTO AGRONÔMICO DO PARANÁ). Cartas climáticas do Paraná, 2014. Disponível em: <http://www.iapar. $\mathrm{br} /$ modules $/$ conteudo/conteudo.php? conteudo $=677>$. Acesso em: 20 jan. 2015.

IBRAFLOR (INSTITUTO BRASILEIRO DE FLORICULTURA). Padrão de qualidade de folhagem de corte Ibraflor, 2014. Disponível em: <http://www.ibraflor.com/publicacoes/ vw.php?cod=74>. Acesso em: 16 jun. 2015.

KIKUCHI, O.Y. Ornamentação floral. São Paulo, Senac, 1995. 73p.

LOBO-GUERRERO, A. Variedades flores y follajes. Colombia: T \& E Flowers, 2009. 28 p.

NAEVE, L.; MIDCAP, J. Preparing cut flowers and houseplants for exhibits. Iowa State University. 2007. 11p. Disponível em: $<$ https:// sheridan.unl.edu/c/document_library/get_file?uuid=47e43acc-8211$4 \mathrm{dfc}-8 \mathrm{e} 1 \mathrm{f}-\mathrm{ce} 1 \mathrm{cda} 162 \mathrm{f} 2 \mathrm{e} \&$ groupId $=135042 \&$.pdf.>. Acesso em: 21 mar. 2014.

OSHIRO, L. et al. Comercialização e produção de folhagem ornamental de corte no Estado de São Paulo. Revista Brasileira de Horticultura Ornamental, v.7, n.1, p.1-8, 2001. Disponível em: <http://rbho.emnuvens.com.br/rbho/article/view/71/8>. Acesso em: 20 jan. 2015.

RIBAS, R.P.; MIGUEL, L. de A. Extração e comercialização de folhagens ornamentais da Mata Atlântica: o caso da verdes (Rumohra adiantiformis) no RS. Revista de Economia e Sociologia Rural, v.42, n.4, p.575-596, 2004. Disponível em: $<$ http://www.scielo.br/pdf/resr/v42n4/24972.pdf>. Acesso em: 20 jan. 2015. doi: 10.1590/S0103-20032004000400003.

SCACE, P.D. The floral artist's guide. Florence: Thomson Delmar Learning, 2001. 288p.

STUMPF, E.R.T. et al. Método para avaliação da potencialidade ornamental de flores e folhagens de corte nativas e não convencionais. Revista Brasileira de Horticultura Ornamental, v.13, p.143-148, 2007. Disponível em: <http://rbho.emnuvens. com.br/rbho/article/view/219/114>. Acesso em: 20 jan. 2015.

STUMPF, E.R.T. et al. Prospecção de plantas nativas do bioma pampa para uso na arte floral. BioScriba, v.1, n.2, p.65-72, 2008. Disponível em: <http://www.bioscriba.org.ar/Stumpfetal2008. pdf>. Acesso em: 20 jan. 2015.

TOGNON, G.B. et al. Ornamental potential and postharvest of Baccharis uncinella D.C. Acta Horticulturae (ISHS), v.1060, p.133-139, 2015. Disponível em: <http://www.actahort.org/ books/1060/1060_18.htm>. Acesso em: 27 jan. 2015. 